GEOMETRICAL PROOF OF TAN A +TAN B + TAN C= TAN A TAN B TAN C.

Similarly, starting from the first of the two following determinants, we obtain

$$
\left|\begin{array}{llll}
a & b & b & b \\
a & b & a & a \\
b & b & a & b \\
a & a & a & b
\end{array}\right|\left|\begin{array}{rrrr}
a & -a & b & -a \\
-b & b & -b & a \\
b & -a & a & -a \\
-b & a & -b & -b
\end{array}\right|=(a-b)^{8} .
$$

In this example the two determinants multiplied are really identical.

Thomas M. MacRobert.
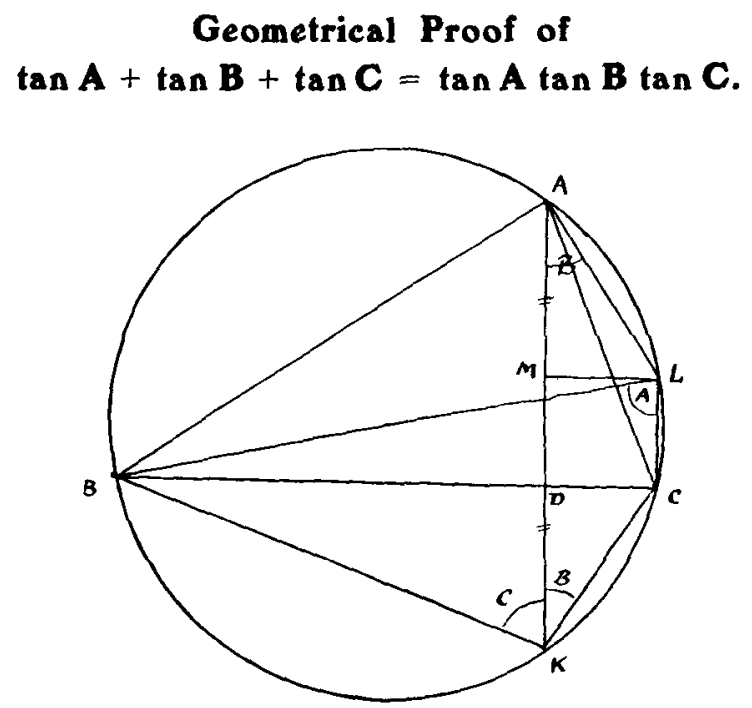

Let $A B C$ be the $\triangle, A D \perp$ to $B C$ produced to meet circumcircle in $K, B L$ a diameter of circumcircle, $L M \perp$ to $A K$. Let $B K, K C$, $C L$ and $L A$ be joined.

$$
\tan A=\frac{a}{C L}, \quad \tan B=\frac{D C}{D K}, \quad \tan C=\frac{D B}{D K} ;
$$

$\therefore \tan A+\tan B+\tan C=\frac{a}{C L}+\frac{a}{D K}=\frac{a(D K+C L)}{C L . D K}$

$$
\tan A \cdot \tan B \cdot \tan C=\frac{a}{C L} \times \frac{D C}{D K} \times \frac{D B}{D K}=\frac{a \cdot A D}{C L \cdot D K},
$$

since

$$
A D . D K=B D . D C \text {. }
$$


Triangles $A M L$ and $K D C$ are duplicates, since $A K$ and $L C$ are parallel chords, and $L M, C D$ perpendicular to them. It follows that $A D=D K+C L$,

$$
\therefore \tan A+\tan B+\tan C=\tan A \cdot \tan B \cdot \tan C .
$$

Colin Kesson.

\section{The Diametric Section Axis of Two Circles.}

Mr Burgess' pretty solution of the problem "to draw a circle cutting three circles diametrically" suggests a question as to the use of sign in treating of co-axial circles.

Any two circles $A$ and $B$ have besides their radical axis an axis with the property that any circle whose centre lies on the axis and which passes through two fixed points cuts the circles at ends of a diameter.

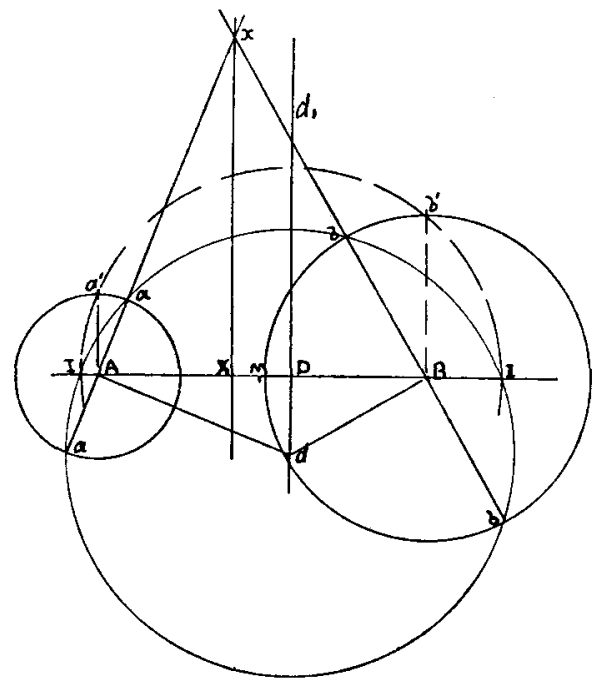

Let $a^{\prime} a^{\prime}, b^{\prime} b^{\prime}$ be diameters at right angles to $A B$, then a circle whose centre $D$ is on $A B$ passes through $a^{\prime} a^{\prime} b^{\prime} b^{\prime}$ and cuts $A B$ at two points $I$ and $l_{1}$, and $d D d_{1}$ at right angles to $A B$ is the required axis.

With $d$ as centre and radius $d I$, construct a circle cutting $B$ at $b$, then $d b=d I$, and $d I^{2}-d B^{2}=D I^{2}-D B^{2}=r_{1}{ }^{2}$, 